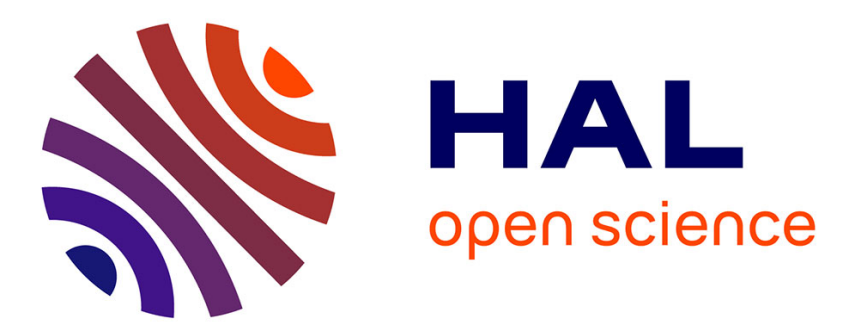

\title{
Caractéristiques psychosociales des adolescents non pratiquants sportifs
}

Julie Boiché, Philippe Sarrazin

\section{To cite this version:}

Julie Boiché, Philippe Sarrazin. Caractéristiques psychosociales des adolescents non pratiquants sportifs. Journal de Pédiatrie et de Puériculture, 2009, 22, pp.62-68. hal-00390121

\section{HAL Id: hal-00390121 \\ https://hal.science/hal-00390121}

Submitted on 31 May 2009

HAL is a multi-disciplinary open access archive for the deposit and dissemination of scientific research documents, whether they are published or not. The documents may come from teaching and research institutions in France or abroad, or from public or private research centers.
L'archive ouverte pluridisciplinaire HAL, est destinée au dépôt et à la diffusion de documents scientifiques de niveau recherche, publiés ou non, émanant des établissements d'enseignement et de recherche français ou étrangers, des laboratoires publics ou privés. 
ADOLESCENTS NON PRATIQUANTS

Julie C.S Boiché ${ }^{1}$ et Philippe G. Sarrazin ${ }^{2}$

$13{ }^{1}$ Laboratoire "Déterminants Interculturels de la Motricité et de la Performance

14 Sportive", Département STAPS, Faculté des Sciences de 1'Homme et de

15 1'Environnement, 117 Rue du Général Ailleret, 97430 Le Tampon, Ile de La Réunion,

16 France.

$17{ }^{2}$ Laboratoire "Sport et Environnement Social”, UFRAPS, BP53, 38041, Grenoble

18 Cédex 9, France.

19

20 Pour toute correspondance relative à cet article merci de vous adresser à Julie Boiché :

210262579568 (téléphone); 0262579571 (fax). E-mail: julie.boiche@univ-reunion.fr

23 Conflits d'intérêts : Aucun 


\section{ADOLESCENTS NON PRATIQUANTS}

\section{Résumé}

2 L'objectif de cette étude était de décrire les caractéristiques psychosociales des

3 adolescents non pratiquants sportifs. 119 adolescents français, scolarisés de la $6^{\text {ème }}$ à la

4 terminale, ont participé à cette étude. Ils ont rempli un questionnaire portant sur les

5 barrières à leur pratique sportive ; leurs motivations à l'égard du sport ; le soutien de

6 leur environnement social ; leur degré d'adhésion aux stéréotypes sexués attachés au

7 sport; et les solutions pour encourager leur pratique. Si leurs réponses n'indiquent pas

8 un profil très « sportif » elles ne révèlent pas non plus une opposition marquée à la

9 pratique sportive. Ils semblent davantage attirés par une pratique physique récréative /

10 d'entretien.

\section{Abstract}

13 The purpose of the present study was to describe some psychosocial characteristics of

14 non sport participant adolescents. 119 French adolescents, pursuing studies from $6^{\text {th }}$ to

$1512^{\text {th }}$ grade, participated to this study. They filled out a questionnaire assessing the

16 perceived barriers to sport participation; their motivations for sport; the perceived social

17 support; their degree of adhesion to gender stereotypes attached to sport; and the

18 solutions that could encourage them to practise an activity. If their answers do not

19 indicate an athletic profile they do not reveal either a frank opposition to sport

20 participation. They seem attracted by a recreational or health oriented practice.

22 Mots-Clés: sédentarité, psychologie, sport.

24 Key-words: sedentary lifestyle, psychology, sport. 
ADOLESCENTS NON PRATIQUANTS

\section{Introduction}

D'après 1'Organisation Mondiale de la Santé, promouvoir l'activité physique (AP)

relève aujourd'hui d'un véritable défi de santé publique, en particulier dans les pays

industrialisés ; la sédentarité pourrait bientôt figurer parmi les 10 principales causes de mortalité et d'incapacités dans le monde [1]. En effet, les effets bénéfiques de 1'AP sont désormais reconnus à bien des points de vue. En premier lieu, l'exercice physique s'est révélé être un facteur préventif de troubles de la santé physique tels que l'obésité [2], le diabète de type II [3] ou encore les maladies cardio-vasculaires [4]. L'AP permet également de prévenir des troubles comme l'anxiété ou la dépression [5] alors que la pratique sportive extra scolaire semble reliée positivement à la réussite académique [67]. Enfin, des études ont montré l'existence d'un lien positif entre la pratique d'une AP et le sentiment d'acceptation sociale [8].

Afin de garantir ces effets positifs, les spécialistes de la santé recommandent généralement d'effectuer environ 30 minutes d'activité modérée par jour, la plupart des jours de la semaine [9]. Ces recommandations sont basées sur des études conduites auprès d'adultes, et le double d'activité - soit 60 minutes quotidiennes - pourrait s'avérer nécessaire pour la santé immédiate et future des jeunes [10]. Malgré plusieurs campagnes de promotion, force est de constater qu'une partie non négligeable des Français de tout âge n'atteignent pas ce seuil à l'heure actuelle. Ce constat a incité les autorités à formuler l'objectif d'une augmentation de $25 \%$ de personnes répondant à ce critère [11]. En effet, la pratique sportive de loisir, à même de compenser un mode de vie de plus en plus sédentaire - caractérisé entre autres par une augmentation sans précédent des transports motorisés [12] - chute de façon continue avec l'âge [13]. Un processus similaire d'abandon particulièrement massif au moment de l'adolescence est rapporté par les fédérations sportives, surtout chez les filles [14-16]. Or, les habitudes 


\section{ADOLESCENTS NON PRATIQUANTS}

1 de pratique développées pendant cette période semblent jouer un rôle important dans

2 l'adoption d'un mode de vie actif à l'âge adulte [17-18].

3 Les adolescents ne pratiquant pas du tout d'activité physique ou sportive

4 constituent donc une population «à risque », qui mérite une attention particulière. Une

5 étude récente indique que près de $14 \%$ des collégiens n'ont jamais pratiqué de sport en

6 dehors du collège [6]. Pourtant, la plupart des études conduites en psychologie portent

7 sur les facteurs d'engagement ou de désengagement sportif [19-21], alors que très peu

8 d'études visent à l'heure actuelle à comprendre l'absence d'engagement. Si quelques

9 travaux se sont penchés sur les caractéristiques psychologiques de populations

10 sédentaires, les échantillons interrogés étaient des adultes, provenant de pays européens

11 autres que la France [22-23].

12 Aussi, l'objectif de cette étude était d'analyser les caractéristiques psycho-sociales

13 de ces adolescents français n'ayant vécu aucune expérience sportive régulière en dehors

14 des cours d'EPS obligatoires, et de cerner leur rapport à ce type de pratique. En premier

15 lieu, nous nous sommes basés sur la littérature existante relativement à la motivation

16 sportive chez les enfants et les adolescents, ainsi qu'aux travaux ayant étudié le

17 désengagement ou l'abandon sportif [19;24-25]. Ce travail nous a permis de lister un

18 certain nombre de barrières constituant des freins potentiels à la pratique dans la

19 population étudiée. Nous avons également relevé un certain nombre de motivations

20 différentes, pouvant être à l'origine d'un engouement pour ce type d'activités.

21 D'autre part, nous nous sommes appuyés sur le modèle Expectation-Valence

22 d'Eccles et ses collaborateurs [26-27]. Ce paradigme, qui fait référence en matière de

23 socialisation dans le domaine de la psychologie, s'intéresse à la façon dont

24 l'environnement social, notamment les parents, contribuent à ce que les enfants et les

25 adolescents valorisent certaines domaines de vie, s'y sentent compétents, et au bout du 


\section{ADOLESCENTS NON PRATIQUANTS}

1 compte, choisissent de s'y investir ou non. Dans cette étude, nous avons retenu trois

2 dimensions de l'influence sociale : les stéréotypes sexués véhiculés quant à la pratique

3 sportive, le soutien des parents, ainsi que celui perçu de la part des pairs.

Finalement, afin d'obtenir des informations susceptibles de guider des

5 interventions visant à promouvoir la pratique chez les adolescents sédentaires, nous

6 avons interrogés les participants sur les solutions à envisager pour les encourager à

7 démarrer la pratique d'une activité sportive régulière.

8 Compte tenu de l'objectif de cette étude, il nous a semblé important d'examiner

9 les effets potentiels du sexe et du niveau d'études sur l'ensemble des variables

10 mesurées.

11 2. Matériel et Méthode

\subsection{Participants et Procédure}

13 Les données de cette étude ont été collectées entre 2003 et 2006 au sein de deux

14 collèges et d'un lycée d'enseignement général de la région de Grenoble, avec la

15 collaboration des équipes administratives et pédagogiques. Après avoir informé les

16 parents des conditions de réalisation de l'étude, plusieurs classes de ces établissements

17 ont reçu la visite de la chercheuse et les élèves volontaires ont rempli un questionnaire.

18 Le critère d'inclusion dans l'échantillon était le suivant : n'avoir jamais pratiqué

19 d'activité sportive régulièrement en dehors des cours d'EPS obligatoires, et ce quel que

20 soit le cadre de la pratique (e.g., club, association, AS). L'échantillon final comporte

21119 adolescents dont les caractéristiques démographiques sont présentées dans le

22 Tableau 1.

\subsection{Mesures}

24 Barrières à la pratique sportive. Sur la base de différents questionnaires et

25 d'études antérieures sur les barrières perçues à l'exercice et les motifs d'abandon sportif 


\section{ADOLESCENTS NON PRATIQUANTS}

$1 \quad$ [19;24-25], une échelle de 9 énoncés a été construite pour les besoins de l'étude (voir

2 Annexe 1). L'intitulé de cette partie du questionnaire était : « A quel point les raisons

3 suivantes correspondent à tes raisons de ne pas faire de sport? ». Les participants

4 disposaient pour répondre d'une échelle de type Likert de 1 («pas du tout d'accord») à

$5 \quad 6$ (《tout à fait d'accord »).

6 Buts poursuivis dans la pratique sportive. La deuxième échelle du questionnaire

7 était destinée à mieux connaître les motivations des participants envers la pratique

8 sportive. A partir des outils développés dans le contexte sportif [28-29] une échelle de

910 énoncés a été créée (voir Annexe 1). L’intitulé de cette partie était : «Dans la partie

10 qui suit, nous te proposons différents buts qui peuvent être poursuivis quand on fait un

11 sport. Pour chacune des phrases, indique à quel point cela pourrait te correspondre ».

12 L'échelle de réponse était là encore une échelle en 6 points.

13 Soutien social perçu. Afin d'estimer le soutien social reçu quant à la pratique

14 sportive, 3 énoncés relatifs aux parents (voir Annexe 1), puis aux amis, ont été

15 proposés, en adaptant l'échelle utilisée dans la théorie Expectation-Valence [26-27]. La

16 consistance interne était satisfaisante pour les parents $(\alpha=.68)$ et les amis $(\alpha=.72)$.

17 Stéréotypes sexués. Une mesure subtile a été utilisée pour estimer le degré

18 d'adhésion aux stéréotypes sexués attachés au sport [30-31]. Des questions portant sur

19 l'utilité et l'importance du sport, ainsi que sur la compétence sportive, ont été posées

20 pour les garçons et les filles, respectivement (voir Annexe 1 pour un exemple d'item).

21 La soustraction entre les deux réponses a été effectuée afin d'examiner le degré

22 d'adhésion aux stéréotypes pro-masculins dans l'échantillon. Plus le score de différence

23 est élevé, plus cela signale 1'adhésion à un stéréotype pro-masculin selon lequel le sport

24 est davantage « une affaire d'homme ». 


\section{ADOLESCENTS NON PRATIQUANTS}

Solutions pour promouvoir la pratique sportive. La dernière partie du

2 questionnaire concernait des solutions envisageables pour encourager ces adolescents à démarrer la pratique d'une activité. Six énoncés étaient proposés en réponse à la question suivante : "Indique pour chacune des propositions suivante, à quel point cela pourrait te faire faire du sport » (voir échelle en Annexe 1). L'échelle de réponse était là aussi une échelle en 6 points (de 1, «pas du tout d'accord 》, à 6, « tout à fait d'accord»).

\subsection{Analyse des Données}

Des analyses descriptives pour chacune des variables mesurées sont présentées dans le Tableau 2. Une série de tests $t$ a ensuite été effectuée pour examiner d'éventuelles différences liées au sexe des participants (voir Tableau 2). Enfin, des MANOVAs ont été conduites afin d'examiner l'effet potentiel du niveau d'études sur les différents groupes de variables psycho-sociales mesurés (voir Tableau 3). Etant donné le faible effectif pour certains niveaux de classe, les participants ont été regroupés selon leur cycle d'étude : «premier cycle » pour les élèves de $6^{\text {ème }}$ et $5^{\text {ème }}$; « deuxième cycle » pour les élèves de $4^{\text {ème }}$ et $3^{\text {ème }}$; « troisième cycle » pour les lycéens. Un résultat significatif a été suivi d'ANOVAs séparées, ainsi que de tests post-hoc de Tuckey.

\section{Résultats et Discussion 3.1. Statistiques descriptives}

Les résultats concernant les barrières perçues vis-à-vis de la pratique sportive indiquent que les principaux obstacles à l'investissement de ces adolescents sont le fait que l'EPS leur suffit, qu'ils perçoivent un manque de temps lié notamment à la charge scolaire, et qu'ils n'ont pas découvert d'activité qui leur plaise réellement ; ces propositions ont reçu un accord relativement important avec une moyenne supérieure au 


\section{ADOLESCENTS NON PRATIQUANTS}

1 milieu de l'échelle de réponse (soit 3,5). Viennent ensuite le manque de goût pour le

2 sport, la faible compétence perçue pour ce type de pratique, et la crainte de se retrouver

3 dans un groupe de pratique peu adapté (i.e., des personnes du même âge mais de

4 meilleur niveau, ou des personnes de même niveau, mais plus jeunes). Les barrières

5 financières, géographiques et de santé, que l'on peut considérer comme les plus

6 rédhibitoires, semblent les moins explicatives de l'absence d'engagement.

7 En admettant que ces participants commencent à pratiquer régulièrement une

8 activité, les buts principalement poursuivis concerneraient des aspects sociaux,

9 ludiques, liés à la santé ou l'apprentissage. En revanche, les buts compétitifs ou de

10 désirabilité sociale reçoivent peu d'écho, avec des réponses en moyenne inférieures à 3.

11 Concernant les perceptions sociales chez ces adolescents, on peut relever des

12 niveaux modérés de soutien social perçu, les parents semblant apporter davantage de

13 support que les amis. D'autre part, les participants semblent en moyenne adhérer

14 modérément aux stéréotypes pro-masculins attachés à l'utilité et l'importance de la

15 pratique sportive, mais de façon plus importance à celui lié à la compétence sportive.

16 De telles croyances ne sont pas anodines puisqu'elles sont corrélées négativement à

17 plusieurs perceptions du soi physique et à la performance sur une tâche motrice chez les

18 filles [31-32].

19 Concernant les solutions à envisager pour encourager ces jeunes à démarrer la

20 pratique régulière d'une activité, le fait de trouver une activité qui leur plaise vraiment,

21 de pouvoir commencer en même temps qu'un(e) ami(e), ou de dégager un espace dans

22 1'emploi du temps semblent les plus prometteuses. Lever les barrières financières ou

23 géographiques, ainsi que recevoir davantage d'encouragements de la part de sa famille,

24 seraient des solutions moins efficaces, puisqu'en moyenne les réponses sont inférieures

25 au milieu de l'échelle de réponse. 
ADOLESCENTS NON PRATIQUANTS

1

2

3

4

\subsection{Effets du sexe}

Concernant les barrières perçues à la pratique sportive, une seule différence significative est apparue. Ainsi, il semble que le manque de temps soit une barrière plus importante pour les garçons $(M=4,19)$ que pour les filles $(M=3,32)$. Concernant les buts poursuivis dans la pratique sportive, une seule différence significative a été observée : les filles valorisent davantage le fait de pratiquer une activité avec un(e) ami(e) $(M=4,50)$, par rapport aux garçons $(M=3,75)$. Aucune différence significative n'est apparue pour les perceptions sociales (soutien social des proches ou stéréotypes sexués). Enfin, parmi les solutions proposées pour promouvoir la pratique, une différence significative est apparue : les filles semblent davantage adhérer à la solution consistant à commencer la pratique d'une activité avec un(e) ami(e) $(\mathrm{M}=4,26)$, par rapport aux garçons $(\mathrm{M}=3,22)$.

\subsection{Effets du niveau d'études}

Les MANOVAs effectuées sur les différents groupes de variables ont révélé un effet significatif du niveau d'études seulement pour les buts poursuivis dans la pratique : Wilk's Lambda 0,638, F $(10,30)=1,574, p<.03$. Les ANOVAs conduites sur les différents buts ont indiqués plusieurs effets simples. Ainsi, le niveau d'études est lié de façon significatif à l'adoption au but d'apprentissage : $F(3,116)=3,314, p<.02$. Le test post-hoc a indiqué que les élèves de premier cycle adhéraient davantage à ce but (M $=4,48)$ par rapport à ceux de troisième cycle $(\mathrm{M}=3,28)$. Un effet est également apparu pour le but de supériorité sociale $: \mathrm{F}(3,116)=2,908, \mathrm{p}<.04$. Là encore, les élèves de premier cycle semblent davantage adhérer à ce but, par rapport à ceux de troisième cycle $(M=2,93$ versus $M=2,06)$.

Un effet simple est également apparu pour le but de désirabilité sociale (i.e., faire plaisir à quelqu'un d'important) : $\mathrm{F}(3,116)=4,017, \mathrm{p}<.01$. Les élèves de premier 


\section{ADOLESCENTS NON PRATIQUANTS}

1 cycle ont obtenu des scores significativement plus élevés $(M=3,17)$ que ceux de

2 second cycle $(\mathrm{M}=2,08)$. Un effet significatif a été observé pour le but de progrès $\mathrm{F}(3$,

$3116)=3,830, p<.01$. Les élèves de premier cycle $(M=4,67)$ se sont cette fois

4 distingués à la fois de ceux de deuxième $(M=3,69)$ et de troisième cycle $(M=3,61)$.

5 Un effet simple a enfin été constaté pour le but compétitif : $F(3,116)=4,323, p<.01$.

6 Les élèves de troisième cycle $(M=1,83)$ ont obtenu des scores significativement plus

7 faibles que ceux de premier $(M=3,14)$ et de deuxième cycle $(M=2,95)$.

\section{Conclusions et Perspectives}

Entre 10 et $15 \%$ des adolescents Français déclarent ne jamais avoir pratiqué

10 d'activité sportive en dehors des cours obligatoires d'EPS. Etant donnés les enjeux de

11 santé attachés à la pratique régulière d'AP, ceux-ci constituent une population à risque.

12 A partir des résultats de cette étude, des perspectives d'actions visant à promouvoir leur

13 engagement dans une activité physique ou sportive régulière peuvent être envisagées.

14 Ainsi, ces jeunes, s'ils ne montrent pas un profil très sportif, sont loin de se déclarer

15 absolument anti-sportifs ; ils semblent privilégier d'autres contextes, comme les études,

16 sont satisfaits de leur pratique sportive dans le cadre scolaire (i.e., l'EPS) et n'ont pas

17 encore découvert d'activité qui les passionne.

18 Dans l'ensemble, leurs réponses reflètent un décalage par rapport à l'offre des

19 fédérations sportives envers ces tranches d'âge. En effet, le système sportif valorise un

20 entraînement de plus en plus régulier et intense en vue de participer à des compétitions

21 voire de préparer au haut niveau [33-34]. Or, ces adolescents semblent peu motivés par

22 les aspects compétitifs, pour privilégier une pratique plutôt récréative ou de santé. Alors

23 que les opportunités de pratique de loisir sont nombreuses pour les enfants, et que

24 1'exercice physique d'entretien constitue une partie importante des pratiques adultes, i1

25 existe peut-être un « fossé » à combler au niveau des adolescents non sportifs. Le fait de 


\section{ADOLESCENTS NON PRATIQUANTS}

1 mettre en place des périodes « portes ouvertes », ou un système de parrainage par un(e)

2 autre adolescent(e) déjà pratiquant, pourraient constituer des pistes pour encourager ces

3 adolescents novices à franchir les portes des clubs dans la lignée des déclarations des

4 participants.

$5 \quad$ Les filles sont très majoritaires dans la population étudiée, et en ce sens, les

6 différences liées au sexe méritent être considérées avec attention. Les résultats indiquent

7 qu'elles se différentient peu des garçons dans leur rapport à la pratique sportive.

8 Toutefois, une différence qui ressort concerne l'importance qu'elles accordent aux

9 relations sociales : le sport semble constituer pour elle un contexte à même d'entretenir

10 leurs amitiés. Cette caractéristique devrait être considérée afin de promouvoir la

11 pratique féminine. De plus, certaines évolutions peuvent être constatées en termes de

12 motivation, au fur et à mesure de l'avancée dans les études. Il semble que si les élèves

13 peuvent être motivés par des aspects variés de la pratique sportive en début de collège,

14 les lycéens se caractérisent par un rejet plus important de 1'apprentissage et de la

15 compétition. Les pratiques proposées devraient donc également prendre en compte le

16 fait qu'avec l'âge, les adolescents sont susceptibles «d'accrocher » de moins en moins à

17 une pratique sportive traditionnelle, et semblent plus attirés par des activités ludiques,

18 récréatives, laissant une part importante aux relations sociales. 


\section{ADOLESCENTS NON PRATIQUANTS}

\section{Références}

[1] Organisation Mondiale de la Santé. La sédentarité, une cause majeure de maladies et d'incapacités. 2002 ; http://www.who.int/mediacentre/news/releases/ release23/fr/

[2] Avenell A, Brown TJ, McGee MA, Campbell MK, Grant AM, Broom J, Jung RT, Smith WCS. What interventions should we add to weight reducing diets in adults with obesity? A systematic review of randomized controlled trials of adding drug therapy, exercise, behavioral therapy or combinations of these interventions. J Hum Nutr Diet 2004;17:293-316.

[3] Smith DW, McFall SL. The relationship of diet and exercise for weight control and the quality of life gap associated with diabetes. J Psychosom Res 2005;59:385-92.

[4] Miller TD, Balady GJ, Fletcher GF. Exercise and its role in the prevention and rehabilitation of cardiovascular disease. An Behav Med, 1997;19:220-9.

[5] Atlantis E, Chow CM, Kirby A, Singh, MF. An effective exercise-based intervention for improving mental health and quality of life measures: a randomized controlled trial. Prev Med 2004;39:424-34.

[6] Laure P, Bisinger C. L'activité physique et sportive régulière : un déterminant des résultats scolaires au collège. Sc Sport. 2008; xx.

[7] Marsh HW, Kleitman S. School athletic participation: Mostly gain with little pain. J Sport Exerc Psych 2003;25:205-228.

[8] Martinsen EW, Stephens T. Exercise and mental health in clinical and freeliving populations. In: Dishman RK, editor. Advances in exercise adherence. Champaign : Human Kinetics; 1994. p. 52-72.

[9] Ministère de la Santé et des Solidarités. Sédentarité et inactivité physique. 2003; http://www.sante.gouv.fr/htm/dossiers/losp/06sedentarite.pdf 


\section{ADOLESCENTS NON PRATIQUANTS}

1 [10] Simon C, Klein C, Wagner A. La sédentarité des enfants et des adolescents: Un enjeu de santé publique. J Pédiatr Puéri 2005;18:217-223.

3 [11] Plan National Nutrition Santé. Activité physique et santé : arguments scientifiques, pistes pratiques. $2005 ;$ http://www.sante.gouv.fr/htm pointsur/nutrition/actions 42 activite. pdf

6 [12] Médiamétrie. 2006 ; http ://www.mediametrie.fr/news.php? rubrique= net \& $\underline{\text { news id }=149}$

8 [13] Ministère de la Jeunesse, des Sports et de la Vie Associative. La France sportive: premiers résultats de 1'enquête “pratiques sportives 2000”. 2001;Stat Info:01-01.

10 [14] Chevalier V. Une population de pratiquants sportifs et leurs parcours : Les 11 cavaliers titulaires d'une licence. Popul 1996;3:573-608.

12 [15] Guillet E. Facteurs et processus de l'abandon sportif : du rôle de l'entraîneur à 13 l'impact des normes culturelles. Une étude longitudinale en handball féminin 14 [thèse]. Grenoble : Université Joseph Fourier ; 2000.

15 [16] Trabal P, Augustini M. L'abandon de la pratique de la boxe française. Actes du 16 VIIème congrès International de 1'Association des Chercheurs en Activités $17 \quad$ Physiques et Sportives, Marseille, 1997.

18 [17] Perkins DF, Jacobs JE, Barber BL, Eccles JS. Childhood and adolescent sports participation as predictors of participation in sports and physical fitness activities

20 during young adulthood. Youth Society 2004;35:495-520.

21 [18] Tammelin T, Nayha S, Hills AP, Jarvelin MR. Adolescent participation in sport and in sports and adult physical activity. Am J Prev Med, 2003;24:1,22-28.

23 [19] Gould D. Understanding attrition in children's sport. In: Gould D, Weiss MR, 24 editors. Advances in pediatric sciences. Champaign, Il: Human Kinetics; 1987. p. 61-85 


\section{ADOLESCENTS NON PRATIQUANTS}

1 [20] Sarrazin PG, Boiché JCS, Pelletier LG. A self-determination theory approach to

2 dropout in athlete. In : Hagger M, Chatzisarandis N, editors. Self-Determination in

3 Exercise and Sport. Champaign : Human Kinetics; 2007. p. 229-241.

4 [21] Sarrazin P, Guillet E. « Mais pourquoi ne se réinscrivent-ils plus ? ». In: Cury F,

5 Sarrazin P, editors. Théories de la motivation et pratiques sportives: Etat des

6 recherches. Paris: PUF; 2001. p. 223-54.

7 [22] Thørgersen-Ntoumani C, Ntoumanis N, Nikitaras N. Typologies of Greek inactive

8 older adults based on reasons for abstaining from exercise and conditions for

$9 \quad$ change. J Sport Sci 2008;26:1341-1350.

10 [23] Vanden Auweele Y, Rzewnicki R, Van Mele V. Reasons for not exercising and

11 exercise intentions: A study of middle-aged sedentary adults. J Sports Sci

$12 \quad 1997 ; 15: 151-165$.

13 [24] Brawley LR, Martin KA, Gyurcsik NC. Problems assessing perceived barriers to

14 exercise: confusing obstacles with attributions and excuse. In: Duda J, editor.

15 Advances in sport and exercise psychology measurement. Morgantown, WV:

16 Fitness Information Technology Inc; 1998. p. 337-50.

17 [25] Weiss M, Chaumeton. Motivational orientations in sport. In: Horn TS, editor.

18 Advances in sport psychology. Champaign : Human Kinetics; 1992. p. 61-99.

19 [26] Eccles JS, Freedman-Doan C, Frome P, Jacobs J, Yoon KS. Gender role

20 socialization in the family: A longitudinal approach. In: Eckes T, Trautner H,

21 editors. The developmental social psychology of gender. Mahwah, NJ: Lawrence

22 Erlbaum Associates; 2000. p. 333-360.

23 [27] Fredricks JA, Eccles JS. Family Socialization, Gender, and Sport Motivation and

24 Involvement. J Sport Exerc Psych 2005;27:3-31 


\section{ADOLESCENTS NON PRATIQUANTS}

1 [28] Brière NM, Vallerand RJ, Blais MR, Pelletier LG. Développement et validation d'une mesure de motivation intrinsèque, extrinsèque et d'amotivation en contexte sportif : L'Échelle de Motivation dans les Sports (EMS). Intern J Sport Psychol $1995 ; 26: 465-89$.

5 [29] Sarrazin P, Biddle S, Famose JP, Cury F, Fox K, Durand M. Goal orientations and

6 conceptions of the nature of sport ability in children: A social cognitive approach.

$7 \quad$ Brit J Soc Psych 1996;35:399-414.

8 [30] Bonnot V, Croizet JC. Stereotype internalization and women's math performance:

9 The role of interference in working memory. J Exp Soc Psych 2007;43:857-66.

10 [31] Chalabaev A, Sarrazin P, Fontayne P. Stereotype endorsement and perceived

11 ability as mediators of the girls' gender orientation-soccer performance relationship.

12 Psych Sport Exerc. 2008; xx.

13 [32] Boiché J, Sarrazin P, Bois J. développement des stéréotypes sexués liés au sport au

14 cours de 1'adolescence et corrélations avec des indicateurs du soi physique : Une

15 étude en contexte naturel. Journées d'Etudes de la Société Française de

16 Psychologie du Sport, Grenoble, 2004. p. 23-24.

17 [33] Brustad RJ, Babkes ML, Smith AL. Youth in sport: Psychological considerations.

18 In: Singer RN, Hausenblas HA, Janelle CM, editors. Handbook of Sport Psychology

19 (2nd Edition). New York: Wiley; 2001. p. 604-635.

20 [34] Lavallee D, Wylleman P, editors. Career transitions in sport: International 21 perspectives. Fitness Information Technology: Morgantown; 2000. 


\section{ADOLESCENTS NON PRATIQUANTS}

$1 \quad$ Annexe 1

2

3 Barrières perçues à la pratique

4 1. Je n'ai pas le temps (trop de devoirs)

5 2. Je n'aime pas le sport

$6 \quad 3$. Je n'ai pas trouvé d'activité qui me plaise

7 4. Ce que je fais en EPS me suffit

8 5. Si je commençais une activité je serais avec des gens plus forts ou plus jeunes que

9 moi

10 6. J'ai des problèmes de santé

11 7. L'activité que je voulais faire coûte trop cher

128 . Je ne suis pas assez bon en sport

13 9. Personne ne peut s'occuper des trajets

15 Buts poursuivis dans la pratique

16 1. Pour m'amuser

17 2. Pour faire une activité avec des ami(e)s

18 3. Pour apprendre des choses

19 4. Pour ne plus me sentir coupable de ne pas faire assez de sport

20 5. Pour me faire de nouveaux-elles ami(e)s

21 6. Pour pouvoir être plus fort(e) que les autres

22 7. Pour faire plaisir à quelqu'un que j'aime beaucoup (parents, ami(e)s)

23 8. Pour avoir la satisfaction de progresser

249 . Pour être plus en forme

25 10. Pour pouvoir gagner des compétitions 


\section{ADOLESCENTS NON PRATIQUANTS}

1 Soutien social reçu de la part des parents

2 1. Mes parents pensent que c'est important de faire du sport

3 2. Mes parents pensent que c'est utile de faire du sport

4 3. Mes parents trouvent que je suis bon-ne en sport

6 Stéréotype sexué relatif à l'utilité du sport

$7 \quad$ A ton avis, à quel point est-ce utile de faire du sport pour les garçons ?

$\begin{array}{llllllll}\text { pas du tout utile } & 1 & 2 & 3 & 4 & 5 & 6 & \text { vraiment très utile }\end{array}$

$9 \quad$ A ton avis, à quel point est-ce utile de faire du sport pour les filles?

$\begin{array}{lllllllll}10 & \text { pas du tout utile } & 1 & 2 & 3 & 4 & 5 & 6 & \text { vraiment très utile }\end{array}$

12 Solutions pour promouvoir la pratique

13 1. Si j'avais plus de temps

14 2. Si j'avais l'argent nécessaire pour payer l'inscription, la licence, le matériel

15 3. Si j'étais plus encouragé(e) par ma famille

16 4. Si je pouvais commencer l'activité en même temps qu'un(e) ami(e)

175 . Si je trouve une activité qui me plaît vraiment

18 6. Si quelqu'un pouvait m'emmener 
ADOLESCENTS NON PRATIQUANTS

Tableau 1: Caractéristiques Démographiques de 1'Echantillon

\begin{tabular}{ccccccccc} 
& \multicolumn{2}{c}{ 1er cycle } & \multicolumn{2}{c}{ 2ème cycle } & & 3ème cycle & Total \\
\hline & 6ème & 5 ème & 4ème & 3ème & 2nde & 1ère & Terminale & \\
\hline Filles & 17 & 15 & 17 & 13 & 12 & 8 & 4 & 86 \\
Garçons & 8 & 2 & 6 & 4 & 4 & 5 & 3 & 32 \\
\hline Total & 25 & 17 & 23 & 17 & 16 & 13 & 7 & 118 \\
\hline
\end{tabular}

Note: l'effectif total est inférieur à l'effectif de l'échantillon car l'un des participants n'a pas indiqué son sexe. 
Tableau 2 : Statistiques Descriptives et Tests t en fonction du sexe

\begin{tabular}{|c|c|c|c|c|c|c|c|}
\hline & \multicolumn{2}{|c|}{ Total $(\mathrm{N}=119)$} & \multicolumn{2}{|c|}{ Garçons $(\mathrm{N}=32)$} & \multicolumn{2}{|c|}{ Filles $(\mathrm{N}=87)$} & \multirow[b]{2}{*}{$p$} \\
\hline & M & ET & M & ET & $\mathbf{M}$ & ET & \\
\hline \multicolumn{8}{|l|}{ Barrières à la pratique } \\
\hline Manque de temps & 3,55 & 1,75 & 4,19 & 1,64 & 3,32 & 1,75 & 0,02 \\
\hline N'aime pas le sport & 3,12 & 2 & 3,09 & 2,13 & 3,13 & 1,97 & 0,94 \\
\hline Pas activité & 3,54 & 1,88 & 3,59 & 1,86 & 3,52 & 1,89 & 0,84 \\
\hline EPS suffisante & 3,93 & 1,73 & 4,26 & 1,67 & 3,82 & 1,74 & 0,22 \\
\hline Trop tard & 2,95 & 1,85 & 3,13 & 1,81 & 2,88 & 1,87 & 0,53 \\
\hline Problèmes de santé & 1,75 & 1,52 & 1,97 & 1,87 & 1,68 & 1,39 & 0,43 \\
\hline Problèmes financiers & 2,32 & 1,82 & 2,50 & 1,95 & 2,25 & 1,77 & 0,51 \\
\hline Faible compétence perçue & 2,98 & 1,72 & 2,72 & 1,82 & 3,08 & 1,69 & 0,31 \\
\hline Problème de trajet & 1,97 & 1,6 & 1,66 & 1,36 & 2,09 & 1,67 & 0,15 \\
\hline \multicolumn{8}{|l|}{ Buts } \\
\hline Amusement & 4,23 & 1,7 & 4,06 & 1,98 & 4,30 & 1,59 & 0,51 \\
\hline Amitié & 4,29 & 1,64 & 3,75 & 1,83 & 4,50 & 1,52 & 0,03 \\
\hline Apprentissage & 3,97 & 1,81 & 3,44 & 2,08 & 4,17 & 1,66 & 0,08 \\
\hline Résoudre culpabilité & 2,77 & 1,77 & 2,47 & 1,80 & 2,88 & 1,76 & 0,26 \\
\hline Rencontre & 3,43 & 1,81 & 3,32 & 1,87 & 3,47 & 1,80 & 0,70 \\
\hline Supériorité sociale & 2,4 & 1,64 & 2,48 & 1,75 & 2,37 & 1,61 & 0,74 \\
\hline Désirabilité sociale & 2,54 & 1,68 & 2,66 & 1,62 & 2,50 & 1,70 & 0,66 \\
\hline Progrès & 4,03 & 1,65 & 3,55 & 1,93 & 4,20 & 1,51 & 0,09 \\
\hline Forme & 4,27 & 1,63 & 3,81 & 1,96 & 4,44 & 1,48 & 0,11 \\
\hline Compétition & 2,67 & 1,85 & 2,69 & 1,94 & 2,67 & 1,83 & 0,96 \\
\hline \multicolumn{8}{|l|}{$\begin{array}{l}\text { Soutien Social } \\
\text { nat }\end{array}$} \\
\hline Parents & 3,9 & 1,25 & 3,88 & 1,42 & 3,91 & 1,18 & 0,91 \\
\hline Amis & 3,6 & 1,26 & 3,77 & 1,26 & 3,54 & 1,26 & 0,40 \\
\hline \multicolumn{8}{|l|}{ Stéréotypes sexués } \\
\hline Importance & 0,46 & 1,89 & 0,38 & 1,93 & 0,49 & 1,89 & 0,77 \\
\hline Utilité & 0,42 & 1,65 & 0,19 & 1,91 & 0,51 & 1,55 & 0,35 \\
\hline Compétence & 0,74 & 1,85 & 0,59 & 2,12 & 0,80 & 1,74 & 0,59 \\
\hline \multicolumn{8}{|l|}{$\begin{array}{l}\text { Solutions } \\
\text { Solution }\end{array}$} \\
\hline Temps & 3,42 & 1,89 & 3,53 & 1,83 & 3,38 & 1,92 & 0,70 \\
\hline Argent & 2,79 & 1,87 & 2,47 & 1,83 & 2,91 & 1,88 & 0,26 \\
\hline Encouragements & 2,31 & 1,7 & 2,34 & 1,73 & 2,30 & 1,69 & 0,90 \\
\hline Démarrer avec un(e) ami(e) & 3,97 & 1,9 & 3,22 & 1,98 & 4,26 & 1,80 & 0,01 \\
\hline Activité & 4,36 & 1,78 & 3,94 & 1,90 & 4,52 & 1,72 & 0,12 \\
\hline Trajet & 2,24 & 1,7 & 2,50 & 1,85 & 2,15 & 1,65 & 0,32 \\
\hline
\end{tabular}


ADOLESCENTS NON PRATIQUANTS

Tableau 3 : Résultats des MANOVAs pour les Effets du Niveau d'Etudes

\begin{tabular}{lccccc} 
& Lambda de Wilks & F & ddl & ddl & $p$ \\
\hline Barrières à la pratique & 0,815 & 0,803 & 9 & 27 & 0,75 \\
Buts & 0,638 & 1,574 & 10 & 30 & 0,03 \\
Soutien Social & 0,927 & 1,400 & 2 & 6 & 0,22 \\
Stéréotypes sexués & 0,934 & 0,848 & 3 & 9 & 0,57 \\
Solutions & 0,838 & 1,108 & 6 & 18 & 0,34 \\
\hline
\end{tabular}

